\title{
Left ventricular assist device may improve glycemic control in diabetes mellitus patients but the reverse is not true
}

\author{
Amer Hamdan ${ }^{1}$, Etienne Puymirat ${ }^{2,3}$, Nicolas Danchin ${ }^{2,3}$, Nadia Aissaoui $^{1,3,4}$ \\ ${ }^{1}$ Department of Intensive Care, Hôpital Européen Georges Pompidou (HEGP) Assistance Publique-Hôpitaux de Paris (APHP), Paris, France; \\ ${ }^{2}$ Department of cardiology, HEGP, APHP, Paris, France; ${ }^{3}$ Université Paris Descartes, Paris, France; ${ }^{4}$ Paris-Cardiovascular-Research-Center, \\ INSERM U970, Paris, France \\ Correspondence to: Nadia Aissaoui, MD, PhD. Department of Intensive Care, Hôpital Européen Georges Pompidou, 20 rue Leblanc, 75015 Paris, \\ France. Email: nadia.aissaoui@ aphp.fr. \\ Comment on: Usoh CO, Sherazi S, Szepietowska B, et al. Influence of Diabetes on Outcomes in Patients After Left Ventricular Assist Device \\ Implantation. Ann Thorac Surg 2018;106:555-60.
}

Submitted Sep 14, 2018. Accepted for publication Sep 19, 2018.

doi: $10.21037 /$ jtd.2018.09.105

View this article at: http://dx.doi.org/10.21037/jtd.2018.09.105

Diabetes mellitus $(\mathrm{DM})$ is common in patients with heart failure (HF). DM is a well-established cardiovascular disease risk factor and a highly prevalent condition, known to cause poor clinical outcomes. In the US, there are currently 23 million patients diagnosed with this disease (1), and 451 million (age, 18-99 years) people with diabetes worldwide (2). This condition has long been established as a cardiovascular disease risk factor, with patients having 4- to 5 -fold increased risk of congestive HF (3).

DM itself has been associated with a 2 -fold increased risk of developing symptomatic congestive HF. In addition, DM is an independent predictor of morbidity and mortality both in symptomatic and asymptomatic $\mathrm{HF}(4,5)$.

The last expert recommendations reported that in patients with HF with reduced ejection fraction, interventions that reduce morbidity and mortality confer similar benefit in the presence or absence of diabetes (6). Thus, the treatment of HF in diabetic patients does not follow specific guidelines. All the drugs used in the routine HF treatment are indicated in the diabetic patients (6).

While heart transplantation is a highly effective therapy for advanced, refractory $\mathrm{HF}$, it is limited to $<10 \%$ of candidates due to a severe shortage of donor organs and a variety of contraindications (6-8). This paucity of effective therapy promoted the development of left VAD, which may be used as (I) bridges to heart transplantation, or (II) a long-term alternative to heart transplantation, also known as destination therapy. LVAD allow to unload the failing ventricle, maintain sufficient end-organ perfusion and improve functional capacity (7).

This could be particularly interesting for the DM patients suffering from advanced $\mathrm{HF}$, as the incidence of diabetes is in continuous increase worldwide and regarding the lack of donors (9).

The impact of DM and glycemic control on clinical outcomes among diabetic patients with advanced HF requiring LVAD support is unclear $(10,11)$.

In this issue of Annals of Thoracic Surgery, Usoh et al. present a detailed analysis of their single-center experience with DM and LVAD outcomes. They aimed to answer to two important questions (12):

(I). Do DM patients have higher mortality and morbidity after LVAD implant compared to nondiabetic patients?

(II). Is there any relationship between the degree of glycemic control and long-term mortality risk in LVAD patients with DM?

The authors have added to the literature evidence that DM is associated with an increased rate of allcause mortality during continuous LVAD support. This retrospective analysis of 191 consecutive adult patients undergoing Heart Mate II LVAD as bridge to transplantation or destination therapy was performed from 2008 to 2014 (12). Although the study experienced all the usual limitations of a retrospective cohort design, including incomplete evaluation of the impact of type 1 versus type 2 
$\mathrm{DM}$, duration of DM, and treatment strategies, these realworld studies can provide valuable insight into optimal patient selection and management practices. DM patients had a more than 2-fold greater risk of death compared to non-DM patients [hazard ratio (HR) $2.28,95 \%$ confidence interval (CI) 1.23 to $4.23, \mathrm{P}=0.009$ ]. These findings are consistent with previous studies (10).

The rate of implantation-associated complications after LVAD insertion is questioned as a reason for this increased mortality tendency seen in the studies in the diabetic populations. In patients undergoing cardiac surgery, diabetes has been identified as an independent predictor for several postoperative outcomes including prolonged ICU-stay, sternal infection, perioperative stroke, and renal dysfunction (13).

Interestingly, there were no significant differences in cumulative rates of infection, neurological dysfunction, renal dysfunction, and rehospitalization between diabetic and non-diabetic patients.

Some studies reported that LVAD implantation was associated with significant improvement in HbA1c, fasting plasma glucose and daily insulin need in DM patients with advanced HF $(14,15)$. These results have some physio-pathologic explanations. Advanced HF, due to poor perfusion, leads to neurohormonal imbalance and poor hemodynamics which in turn worsen glycemic control by increasing insulin resistance. Implantation of a LVAD restores hemodynamics with improved cardiac output and index and results in better DM control by reducing adrenergic stimulation, improving mesenteric/ skeletal perfusion and increasing physical activity. These improvements may allow reducing the usual post-surgical cardiac surgery (13).

Anyway, Usoh et al. reported that despite no increased LVAD-associated complications in diabetic patients when compared with patients not suffering from diabetes, the occurrence of an initial complication (including infection, rehospitalization, or neurological dysfunction) led to higher mortality in the diabetic patient group (12).

This leads us to concluding that the increased mortality does not necessarily imply a worse performance of LVADs in the diabetic population, but could indicate an already more severe disease course in these patients, prone to have more than one comorbid condition.

Understanding the mechanisms of the increased mortality may permit to reduce it. It would allow identifying which patient with DM is at the highest risk of death and would avoid such invasive therapies needing cardiac surgery and invasive ventilation. One objective of the authors was to assess the influence of glycemic control on probability of 3-year death after LVAD-implantation, comparing well controlled pre-LVAD diabetes (HbA1c $<7.0 \%$ ) and uncontrolled pre-LVAD (HbA1c) (12). The authors report that the cumulative probability of death was $48 \%$ in uncontrolled diabetics and $30 \%$ in well controlled diabetics but it does not reach the significance (overall $\log$-rank $\mathrm{P}=0.193)$. Absence of evidence of an effect is not evidence of absence of effect. Also, DM $<7.0 \%$ did not have a significantly increased risk compared to non-DM: HR 1.49, 0.60-3.70; achieving adequate DM control before LVAD implantation should be sought. That negative last result may be explained by the fact that $\mathrm{HbA1c}$ reflects the average blood sugar levels over a period of 3 months. The rate of hbAlc does not allow assessing the duration of DM especially in the last phases of advanced HF when the patients have often cachexia (6).

Of note, the inclusion criteria defined DM patients as Pre-LVAD implantation HbA1c (HbA1c) $\geq 6.5 \%$ whereas the glycemic control was defined around $7 \%$.

In conclusion, diabetes is a highly prevalent comorbid condition in HF patients, with increasing incidence worldwide. Improvements in technology, especially the advent of smaller, durable continuous flow pumps, have led to use of LVADs in a much broader population of patients in the last 10 years. These findings show that the use of LVADs in this population is associated with an increased long-term mortality following device implantation. The potential reasons toward this pattern have not been clearly identified and this study failed to show an influence of the middleterm glycemic control prior to LVAD support on long-term mortality. New larger studies are needed to understand the mechanisms in order to reduce this unacceptable mortality. Meanwhile, while the clinician assesses the candidacy of diabetic patients for LVAD implantation, DM should be included in weighing benefits and risks and seen as a mortality risk in addition to the cardiovascular risk.

\section{Acknowledgements}

None.

\section{Footnote}

Conflicts of Interest: N Aissaoui: Medtronic, Thoratec, Abiomed. Other authors have no conflicts of interest to declare. 


\section{References}

1. Bullard KM, Cowie CC, Lessem SE, et al. Prevalence of Diagnosed Diabetes in Adults by Diabetes Type United States, 2016. MMWR Morb Mortal Wkly Rep 2018;67:359-61.

2. Cho NH, Shaw JE, Karuranga S, et al. IDF Diabetes Atlas: Global estimates of diabetes prevalence for 2017 and projections for 2045. Diabetes Res Clin Pract 2018;138:271-81.

3. Kannel WB, Hjortland M, Castelli WP. Role of diabetes in congestive heart failure: the Framingham study. Am J Cardiol 1974;34:29-34.

4. Shindler DM, Kostis JB, Yusuf S, et al. Diabetes mellitus, a predictor of morbidity and mortality in the Studies of Left Ventricular Dysfunction (SOLVD) Trials and Registry. Am J Cardiol 1996;77:1017-20.

5. Swan JW, Anker SD, Walton C, et al. Insulin resistance in chronic heart failure: relation to severity and etiology of heart failure. J Am Coll Cardiol 1997;30:527-32.

6. Ponikowski P, Voors AA, Anker SD, et al. ESC Scientific Document Group2016 ESC Guidelines for the diagnosis and treatment of acute and chronic heart failure: The Task Force for the diagnosis and treatment of acute and chronic heart failure of the European Society of Cardiology (ESC) Developed with the special contribution of the Heart Failure Association (HFA) of the ESC. Eur Heart J 2016;37:2129-200.

7. Aissaoui N, Jouan J, Gourjault $M$ et al. Understanding Left Ventricular Assist Devices. Blood Purif 2018;46:292-300.

8. Aissaoui N, Morshuis M, Maoulida H, et al. Management

Cite this article as: Hamdan A, Puymirat E, Danchin N, Aissaoui N. Left ventricular assist device may improve glycemic control in diabetes mellitus patients but the reverse is not true. J Thorac Dis 2018;10(Suppl 33):S4093-S4095. doi: 10.21037/ jtd.2018.09.105 of end-stage heart failure patients with or without ventricular assist device: an observational comparison of clinical and economic outcomes. Eur J Cardiothorac Surg 2018;53:170-7.

9. Wild S, Roglic G, Green A, et al. Global prevalence of diabetes: Estimates for the year 2000 and projections for 2030. Diabetes Care 2004;27:1047-53.

10. Asleh R, Briasoulis A, Schettle SD, et al. Impact of Diabetes Mellitus on Outcomes in Patients Supported With Left Ventricular Assist Devices: A Single Institutional 9-Year Experience. Circ Heart Failure. 2017;10. doi: 10.1161/CIRCHEARTFAILURE.117.004213.

11. Young JB, Vest AR. Is There a Sweet Spot for Left Ventricular Assist Devices and Diabetes Mellitus? Circ Heart Fail 2017;10.

12. Usoh CO, Sherazi S, Szepietowska B, et al. Influence of Diabetes on Outcomes in Patients After Left Ventricular Assist Device Implantation. Ann Thorac Surg 2018;106:555-60.

13. Bucerius J, Gummert JF, Walther T, et al. Impact of diabetes mellitus on cardiac surgery outcome. Thorac Cardiovasc Surg 2003;51:11-6.

14. Choudhary N, Chen L, Kotyra L, et al. Improvement in glycemic control after left ventricular assist device implantation in advanced heart failure patients with diabetes mellitus. ASAIO J 2014;60:675-80.

15. Patel N, Gluck JA, Radojevic J, et al. Left ventricular assist device implantation improves glycaemic control: a systematic review and meta-analysis. ESC Heart Fail 2018. [Epub ahead of print]. 